\title{
DYNAMICS OF LEAN PREMIXED FLAMES STABILIZED ON A MESO-SCALE BLUFF-BODY IN AN UNCONFINED FLOW FIELD
}

\author{
Yu JEONG KIM ${ }^{1}$, BoK JiK LEE ${ }^{2}$ AND Hong G. IM ${ }^{1, *}$
}

\begin{abstract}
Two-dimensional direct numerical simulations were conducted to investigate the dynamics of lean premixed flames stabilized on a meso-scale bluff-body in hydrogen-air and syngas-air mixtures. To eliminate the flow confinement effect due to the narrow channel, a larger domain size at twenty times the bluff-body dimension was used in the new simulations. Flame/flow dynamics were examined as the mean inflow velocity is incrementally raised until blow-off occurs. As the mean inflow velocity is increased, several distinct modes in the flame shape and fluctuation patterns were observed. In contrast to our previous study with a narrow channel, the onset of local extinction was observed during the asymmetric vortex shedding mode. Consequently, the flame stabilization and blow-off behavior was found to be dictated by the combined effects of the hot product gas pocket entrained into the extinction zone and the ability to auto-ignite the mixture within the given residence time corresponding to the lateral flame fluctuations. A proper time scale analysis is attempted to characterize the flame blow-off mechanism, which turns out to be consistent with the classic theory of Zukoski and Marble.
\end{abstract}

Mathematics Subject Classification. 80A25, 76F65, 76D05, 65L06, 80A32, 76B47, 76N15

Received March 28, 2018. Accepted July 18, 2018.

\section{INTRODUCTION}

Understanding of the mechanism of flame stabilization and blow-off processes has been one of the most critical subjects in premixed combustion applications, such as gas turbine engines and propulsion systems, in which a bluff-body is routinely used as a flame holder. Experimental $[4,17,18]$ and numerical [5-7] studies have been conducted to understand the physical mechanisms to predict the combustion stability under a wide operating conditions. However, due to the complexities and many physical and chemical parameters involved, such as the bluff-body shape, size, flow field and mixture compositions, it is difficult to obtain comprehensive understanding of the phenomena. Kedia et al. $[6,7]$ have undertaken a series of detailed analysis to investigate the effects of the heat losses to the bluff-body on the blow-off characteristics. In these studies, the blow-off conditions were reached for lean mixtures near the flammability limit at lower flow velocities. For the mixture at richer compositions, the blow-off occurs at higher velocities, at which more complex flow dynamics are coupled to the flame chemistry in the recirculation region. The mechanisms for blow-off at such conditions become

Keywords and phrases: Direct numerical simulation, Flame dynamics, Bluff-body stabilization, Premixed flames, Blow-off.

${ }^{1}$ Clean Combustion Research Center, King Abdullah University of Science and Technology (KAUST), Thuwal 23955-6900, Saudi Arabia.

2 School of Mechanical Engineering, Gwangju Institute of Science and Technology (GIST), Gwangju 61005, Republic of Korea.

* Corresponding author: hong.im@kaust.edu.sa 
significantly different, often exhibiting highly transient patterns of extinction and re-ignition, as discussed in a recent review paper by Shanbhugue et al. [16]. The present study aims to shed more insights into the latter cases.

Shanbhugue et al. [16] summarized previous studies of the lean blow-off dynamics behind the bluff-body, and reported that blow-off occurs in multiple steps starting with local extinction along the flame sheet, and the ultimate onset of the final blow-off is attributed to the cooling and shrinking of the wake region. An early theoretical work proposed the blow-off criterion based on the heat exchange balance between heat supplied by the hot recirculating flow and the heat released by the ignition of reactants [3]. Zukoski and Marble [23] proposed a simple Damköhler number correlation between the flow contact time with the wake and a critical ignition time at the blow-off condition. An experimental study by Williams et al. [20] stated with a similar view: "It is postulated that flame blows out when the eddy region regains less heat from the newly ignited gases than is required to ignite these gases." A theoretical analysis by Kundu et al. [11] also reported that the recirculation zone length is an important parameter to control stability and to determine blow-off velocity based on the heat exchange to the combustible stream.

Recently, Lee et al. $[12,13]$ conducted high-fidelity numerical simulations to investigate the blow-off dynamics of bluff-body-stabilized flames in a narrow channel with a height of $1 \mathrm{~mm}$. Although the domain of the channel was at a miniature scale for computational cost consideration, the simulations revealed complex transient events that provide general insights into the fundamental mechanisms. There were three key findings: the onset of local extinction is the most critical factor to determine the total extinction condition, the observed periodic local extinction and recovery phenomenon is controlled by re-ignition of the mixture near the recirculation zone, and the remaining shortened flame attached to the bluff-body is more robust for the syngas/air flame than the hydrogen flame because its heat release is less sensitive to the temperature fluctuations. Consequently, the blow-off phenomenon was considered an extinction process determined by the first onset of the first quenching event in the recirculation zone. The Damköhler number based on the local extinction condition was found to correlate well with the blow-off limits.

While the high fidelity simulations provided valuable insights, whether the findings of these studies are directly applicable to practical large systems must be assessed carefully. In particular, the flow confinement within the narrow flow passages between the two channel walls may affect the flame and wake dynamics behind the bluffbody, which would be different in large scale systems. Therefore, it is necessary to extend the investigation of the flame dynamics by suppressing the confinement effects.

The present study thus aims to address this issue by conducting simulations with a larger domain size to minimize the narrow channel effects. As in the previous studies $[12,13]$ the flame/wake dynamics of lean premixed hydrogen-air and syngas-air mixtures is investigated in response to different inflow velocities. Flame blow-off condition was reached by increasing the inflow velocity at fixed mixture composition, typically above the flammability limit. As will be shown in the results, some significant differences in the flame behavior, including different types of vortex shedding modes, which subsequently affects the key controlling mechanisms for the flame blow-off. The objective of this study is to understand what governs the blow-off limit of bluff-bodystabilized flames under a weakly confined channel. Following the previous studies, the characteristics time scales and the corresponding quantities are discussed to identify the characteristics of local extinction and blow-off event.

\section{Configuration AND NUMERICAL METHOdS}

Direct numerical simulations (DNS) were carried out to solve fully compressible multi-species reactive NavierStokes equations with a finite difference method of eighth order in space and fourth order in time [2]. Lean premixed flames of hydrogen-air and syngas-air $\left(\mathrm{H}_{2}\right.$ to $\mathrm{CO}$ ratio of 1$)$ mixtures with an equivalence ratio $(\Phi)$ of 0.5 were investigated. For both mixture cases, the inflow condition was set at temperature of $300 \mathrm{~K}$ and pressure of $1 \mathrm{~atm}$. The corresponding density ratios $\left(\rho_{\mathrm{u}} / \rho_{\mathrm{b}}\right)$ between reactant and product are 4.91 for hydrogenair and 5.15 for syngas-air, respectively, determined based on the one-dimensional freely propagating premixed flame. Detailed kinetics mechanisms, consisting of 9 species and 19 reactions [14] for hydrogen-air mixture, 


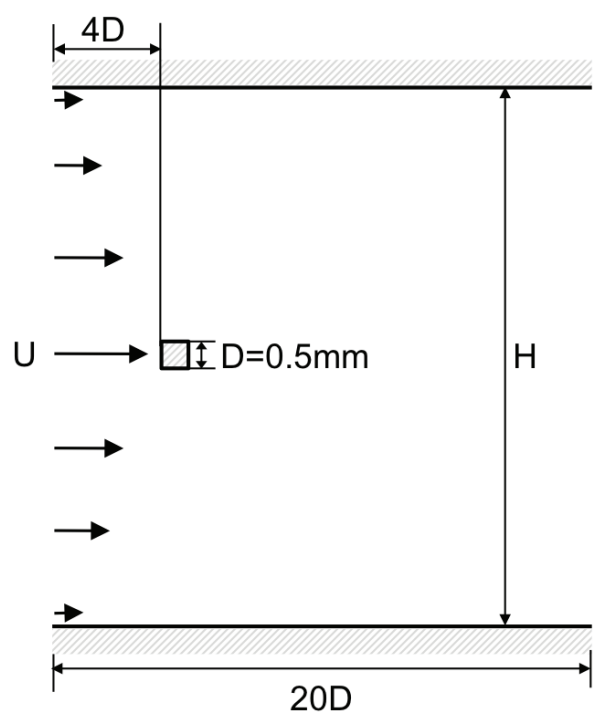

Figure 1. Configuration with dimensions of a two-dimensional channel with a square bluffbody flame holder.

and 12 species and 33 reactions [15] for syngas-air mixture were used. Chemical kinetics, thermodynamics, and transport properties were evaluated using CHEMKIN libraries [8, 9], and the mixture-averaged species diffusion model was used.

A two-dimensional channel of $10 \mathrm{~mm}$ by $10 \mathrm{~mm}$ was used as a computational domain, as shown in Figure 1. A bluff-body flame stabilizer was represented by a square cylinder of $0.5 \mathrm{~mm}(D)$, whose leading edge is located at $2 \mathrm{~mm}(4 D)$ downstream of the inflow boundary. The corresponding blockage ratio $(\beta=D / H)$ is 0.05 , which is ten times lager than that in our previous studies $[12,13]$. The domain size $(H)$ was chosen to minimize the confinement effect due to the narrow channel between the solid walls. The test was done for different blockage ratios $(\beta)$ until a convergence was reached for the Strouhal number $(S t=f D / U)$ of the vortex shedding for the non-reacting condition [10].

A Cartesian grid system with a uniform spacing of $\Delta x=\Delta y=10 \mu \mathrm{m}$ was chosen based on the resolution requirement for the corresponding reference laminar flame structure after the grid convergence tests with the size of $2.5,5,10$, and $12.5 \mu \mathrm{m}$. A square bluff-body stabilizer was modeled as a union of logical MPI blocks and excluded from the computing, imposing non-communicating physical boundary conditions along the embedded boundaries. A typical run with a Cray XC40 cluster (Shaheen II) for time integration up to $10 \mathrm{~ms}$ took approximately 8800 and $16000 \mathrm{CPU}$ hours, for hydrogen-air and syngas-air mixture, respectively. The simulations were conducted longer than 40 flow-through times, which was sufficient to eliminate the transient characteristics and to reach a stationary or limit cycle behavior. No-slip and adiabatic conditions were applied to the channel walls and bluff-body surfaces. For the boundary conditions for the channel walls and bluff-body, the isothermal boundary condition of channel wall at $T_{\text {wall }}=298 \mathrm{~K}$ was also considered in the previous study [13], in which no discernible differences were found in flame dynamics. Moreover, additional simulations were conducted with the isothermal boundary condition of the bluff-body at $T=600 \mathrm{~K}$, and for the conditions under study the overall flame dynamics during the blow-off event was unaffected. Therefore, only the results with the adiabatic boundary conditions are reported here. Non-reflecting characteristic boundary conditions were applied to the inflow and outflow boundaries [21,22]. A fully developed parabolic velocity profile with a mean value of inflow velocity $(U)$ was imposed at the inflow boundary. For parametric studies at each mixture condition, the mean inflow velocity was increased smoothly in a quasi steady-state manner from a previous value to a target value, and was then kept constant until the limit cycle was reached, after which the dynamic response of the flames at the given inflow velocity was investigated. 

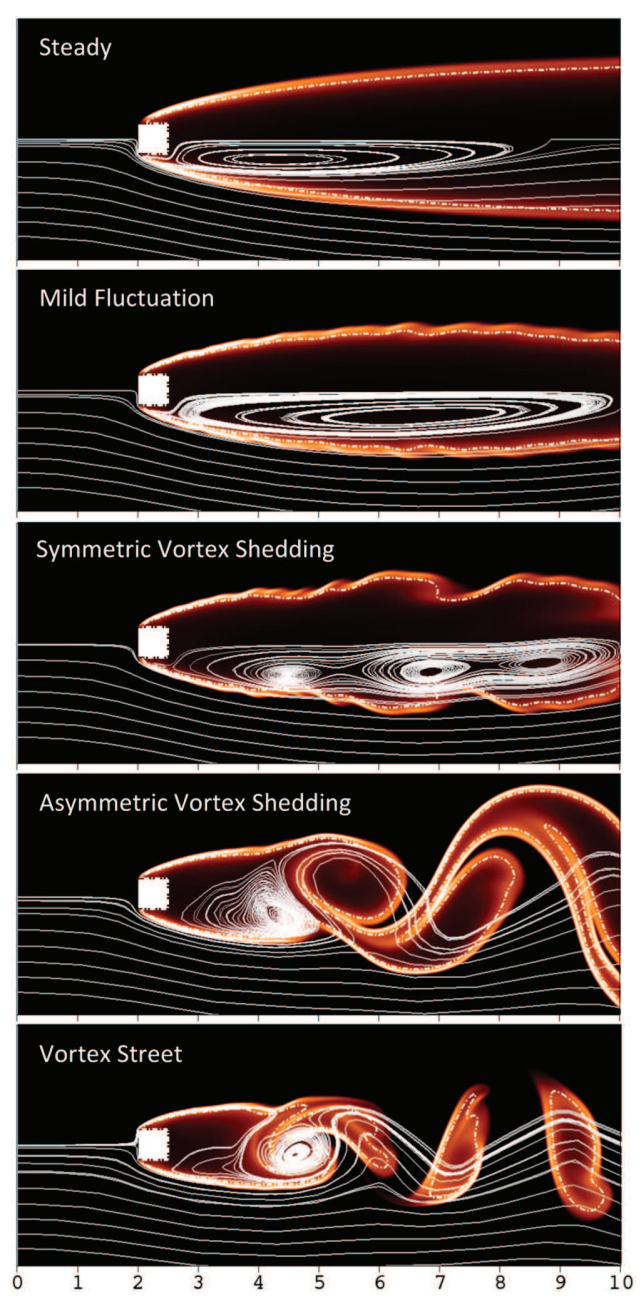

FIGURE 2. Isocontours of heat release rate for hydrogen-air flames illustrating the five different flame modes. Streamlines are overlaid.

\section{Results}

Reacting flow dynamics were investigated for both lean premixed hydrogen-air and syngas-air flames stabilized on a bluff-body by incrementally ramping up the mean inflow velocity $(U)$ until the ultimate blow-off occurs. As the mean inflow velocity increases, distinct modes of flame behavior were observed, such as steady without fluctuations (steady), mild fluctuation (MF), symmetric vortex shedding (SVS), asymmetric vortex shedding (AVS), and vortex street (VST) mode. These are visualized in Figure 2 with partial streamlines. At some critical velocities, the flame exhibits a transient event of the combination of several flame modes. All of these flame modes were observed in both hydrogen-air and syngas-air mixtures. The following analysis is divided into two parts. The first part is the general chemical effects between hydrogen-air and syngas-air mixtures. The results are compared with our previous studies $[12,13]$ in order to understand distinct characteristics of the flame blow-off in the absence of the flow confinement effect. Subsequently, the mechanism of blow-off is analyzed by assessing various characteristic time scales, thereby identifying the key relevant physical and chemical subprocesses that are responsible for the blow-off conditions. 
(a)

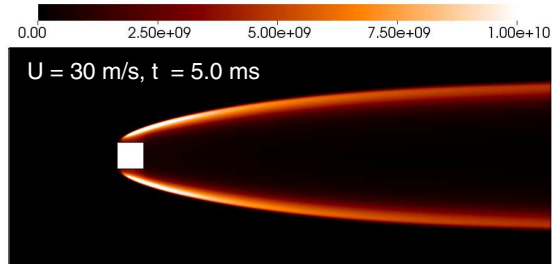

(b)

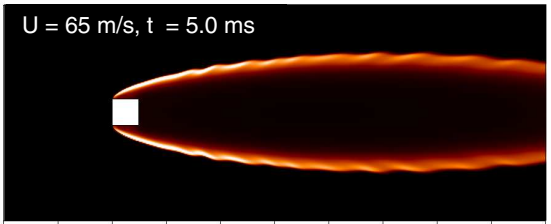

(c)

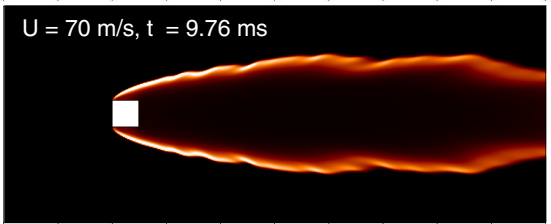

(d)

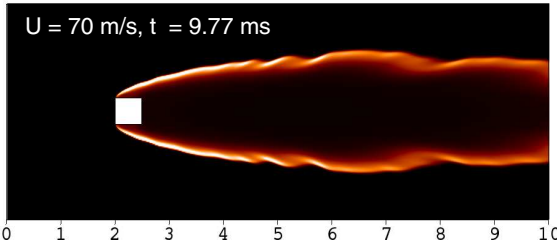

FiguRE 3. Isocontours of heat release rate for hydrogen-air flames: (a) $U=30 \mathrm{~m} / \mathrm{s}$, (b) $U=$ $65 \mathrm{~m} / \mathrm{s}$, and $(\mathrm{c}, \mathrm{d}) U=70 \mathrm{~m} / \mathrm{s}$. Streamlines are overlaid.

\subsection{Chemical effects: hydrogen-air vs. syngas-air}

Flame dynamics for hydrogen-air and syngas-air mixtures, observed from the steadily stable mode to blow-off as the inflow velocity was ramped up to the blow-off limit, is described in detail. Comparison between the two mixtures and between highly confined and less-confined conditions is presented.

\subsubsection{Hydrogen-air flames}

Figure 3 shows instantaneous snapshots of heat release rate at (a) $U=30 \mathrm{~m} / \mathrm{s}$, (b) $U=65 \mathrm{~m} / \mathrm{s}$, and (c, d) $U=70 \mathrm{~m} / \mathrm{s}$. Until $U=30 \mathrm{~m} / \mathrm{s}$, flame is symmetrically anchored near the leading edge of bluff-body without any fluctuations. Due to the absence of the flow confinement, the recirculating flow reaches the separating flow on the leading edge and is generated on the top and bottom of the bluff-body, allowing the flame to stabilize on the leading edge of the bluff-body, unlike our previous studies $[12,13]$. Isocontours of the heat release rate with the maximum level at $10^{10} \mathrm{~W} / \mathrm{m}^{3}$ are used to depict various modes of flame dynamics. Recirculation zones are generated behind the bluff-body, extended to nearly the entire domain length. The distance between two attached flames are also wider than the previous studies $[12,13]$ due to the absence of the confinement. When the inflow velocity is increased to $U=35 \mathrm{~m} / \mathrm{s}$, small scale fluctuations, referred to as the MFs, start to develop on the shear layers and subsequently convected downstream along the flame front. As the velocity is further raised, the amplitude of the MF increases until $U=65 \mathrm{~m} / \mathrm{s}$, while the distance between the two flames becomes narrower towards the downstream according to the reduced size of the recirculation zone. At a higher velocity of $U=70 \mathrm{~m} / \mathrm{s}$, symmetrical fluctuations at a larger scale, called the SVS, are observed with a frequency of 24 $\mathrm{kHz}$. The length of the recirculation zone behind the bluff-body periodically contracts and expands so that the curved flame structures continue to be generated and convected downstream, as shown in Figures 3c and 3d. 

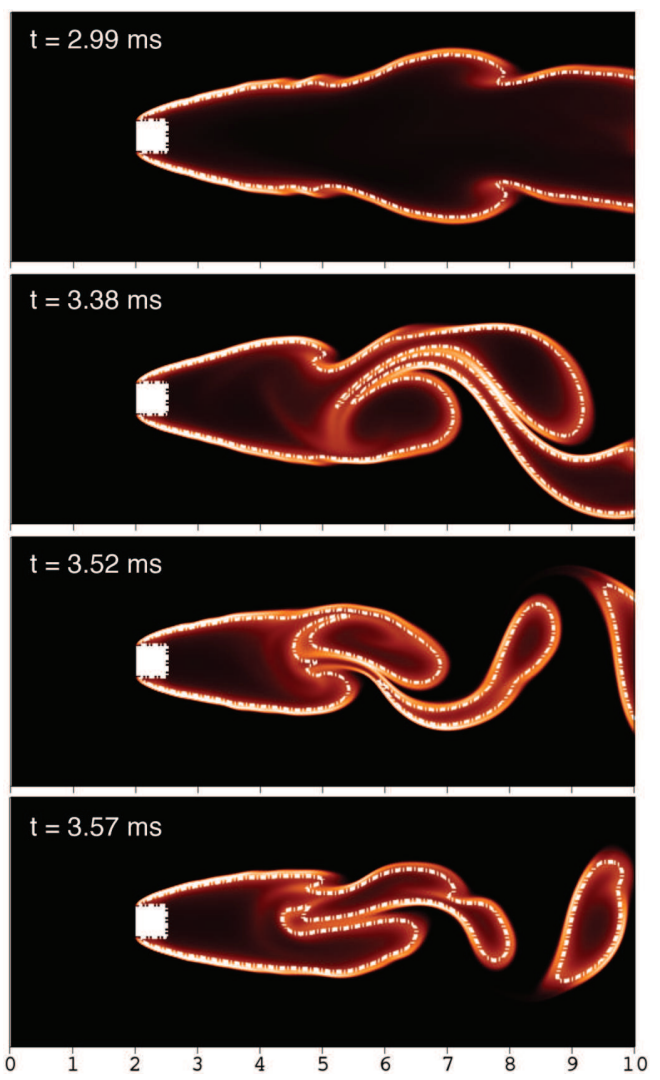

FiguRE 4. Isocontours of heat release rate with contourlines of progress variable based on water mass fraction at 0.5 for hydrogen-air flames at $U=75 \mathrm{~m} / \mathrm{s}$.

This behavior was also observed in an experimental study [1] with a larger scale system, while such behavior was not observed in our previous numerical studies $[12,13]$ with a narrow channel configuration, due to the confinement effect which retained a short recirculation zone.

At $U=75 \mathrm{~m} / \mathrm{s}\left(R e_{D}=1990\right)$, the flame starts to undergo asymmetrical fluctuations and transitional behavior. Figure 4 shows a sequence of transitions exhibiting the three different patterns from the SVS, AVS, and finally the VST mode. Starting from SVS, AVS emerges from the downstream region and moves upstream, eventually leading to local extinction, as shown in the snapshots at $t=3.52 \mathrm{~ms}$. Subsequently, a transition to VST mode which lasts only for a short period $(0.02 \mathrm{~ms})$ at this inflow velocity. When the local extinction occurs, a hot product zone is isolated in a small area behind the bluff-body. Due to the reduced confinement effects in the present study, the hot recirculation zones behind the bluff-body is allowed a wider lateral movement as shown by the AVS mode. After a short period of the VST mode, the SVS mode is recovered and the SVS-AVS-VST cycle repeats. The onset of local extinction is found at the entrainment point of the fresh mixture in the AVS mode (at $t=3.52 \mathrm{~ms}$ in Fig. 4). Lateral fluctuations in AVS mode are observed at a frequency of about $25 \mathrm{kHz}$.

During the AVS mode, the location of the local extinction was chosen according to the progress variable analysis over the entire duration of the AVS mode. Figure 5 shows the corresponding temporal profiles of the progress variable, defined by the normalized water mass fraction, along the central axis from the trailing edge $(x=2.5 \mathrm{~mm})$. Various times were chosen after a full limit cycle behavior is achieved. The result at $t=3.52 \mathrm{~ms}$ (shown in Fig. 4) is also plotted. The local extinction points are chosen at the first point of progress variable crossing below 0.5. Despite the large unsteady fluctuations due to the AVSs, the local extinction points are located remarkably at a fixed location around $x=5 \mathrm{~mm}$. 


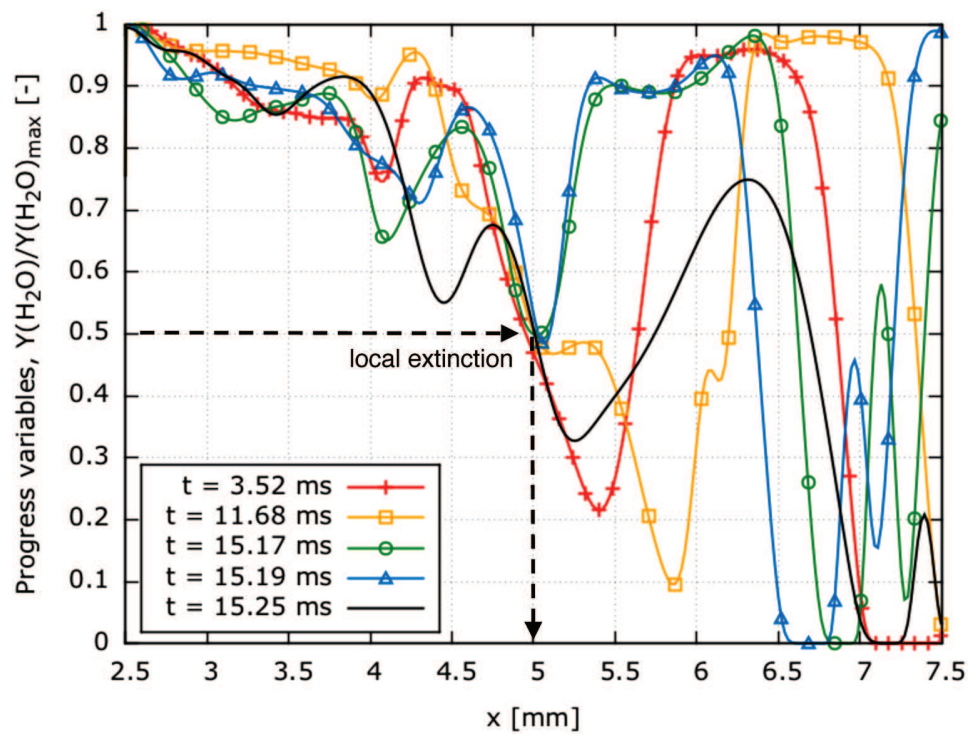

Figure 5. Temporal evolution of axial progress variables for hydrogen-air flames at $U=75 \mathrm{~m} / \mathrm{s}$.

The temporal evolution of heat release rate for the $U=75 \mathrm{~m} / \mathrm{s}$ case is shown in Figure 6 . The black line denotes the heat release rate integrated over the entire computational domain, and the red and blue lines are partially integrated values in the upstream $(2-5 \mathrm{~mm})$ and downstream $(5-10 \mathrm{~mm})$ of the local extinction point, respectively, where the former represent the heat release rate in the hot recirculation zone. Comparing the three curves, it is evident that the large fluctuations in the total heat release rate is mainly attributed to the flame surface area generation (by flame wrinkling) and destruction (by local extinction). It is also seen that the peaks in the heat release rate in the anchored flame region (red line) follows the peaks in the other curves, implying that the local extinction events leads to the entrainment of reactants to the upstream recirculation zone, resulting in an increased heat release rate.

Figure 7 shows the flame images as the mean inflow velocity is further increased to $U=80$ and $85 \mathrm{~m} / \mathrm{s}$ $\left(R e_{D}=2120\right.$ and 2260, respectively). Similar transitions of the SVS-AVS-VST mode cycle are observed, albeit with a longer residence time for the VST mode compare to that for $U=75 \mathrm{~m} / \mathrm{s}$. During the VST mode, periodic lateral fluctuations of the flame in the hot recirculation zone are observed at a frequency of about $33 \mathrm{kHz}$, and then the flame regrows and recover its symmetry back to SVS mode. The level of fluctuations are significantly increased, and the flame blow-off and total extinction occurs at a slightly higher inflow velocity of $U=85.2 \mathrm{~m} / \mathrm{s}$.

Figure 8 shows a sequence of snapshot images during the blow-off event from the highly unstable state to total extinction $\left(R e_{D}=2260\right)$. The flame undergoes a few cycles of periodic transitions, and when the asymmetric shedding behind the bluff-body becomes too strong for the attached flame kernel to survive, the blow-off results. The residence time for the VST mode is longer than that of $U=75 \mathrm{~m} / \mathrm{s}$. The longest and the shortest residence times for the VST mode are 1.08 and $0.22 \mathrm{~ms}$, respectively, at this velocity. In addition, the residence time for AVS and VST modes is longer than symmetric shedding (SVS) (less than $0.17 \mathrm{~ms}$ ). That is, AVS-VST modes become dominant near the blow-off limit. The near-blow-off dynamics signified with the asymmetric shedding behavior is consistent with experimental observations [1, 19].

\subsubsection{Syngas-air flames}

For the syngas-air mixture, general observations are similar to those for the hydrogen-air flames. Figure 9 presents the instantaneous snapshots of the heat release rate with the maximum level at $5 \times 10^{9} \mathrm{~W} / \mathrm{m}^{3}$ : (a) $U=20 \mathrm{~m} / \mathrm{s}$, (b) $U=40 \mathrm{~m} / \mathrm{s}$, and (c, d) $U=48.6 \mathrm{~m} / \mathrm{s}$. At low-velocity ranges, flames are stabilized on the 


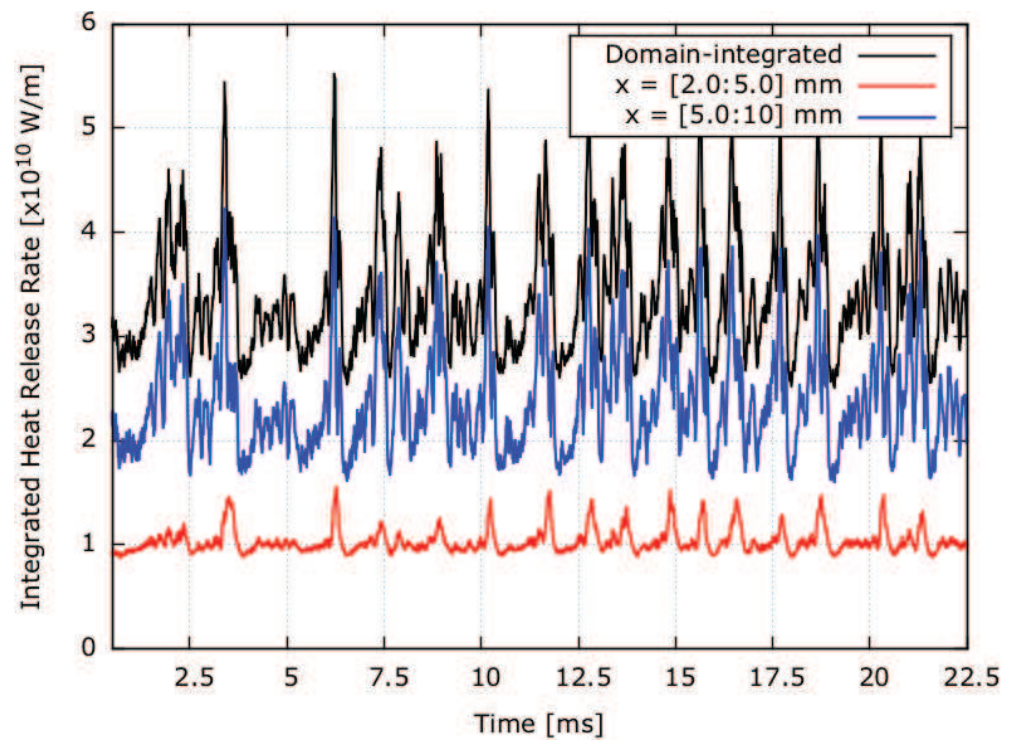

FiguRE 6. Temporal evolution of axial progress variables for hydrogen-air flames at $U=75 \mathrm{~m} / \mathrm{s}$.
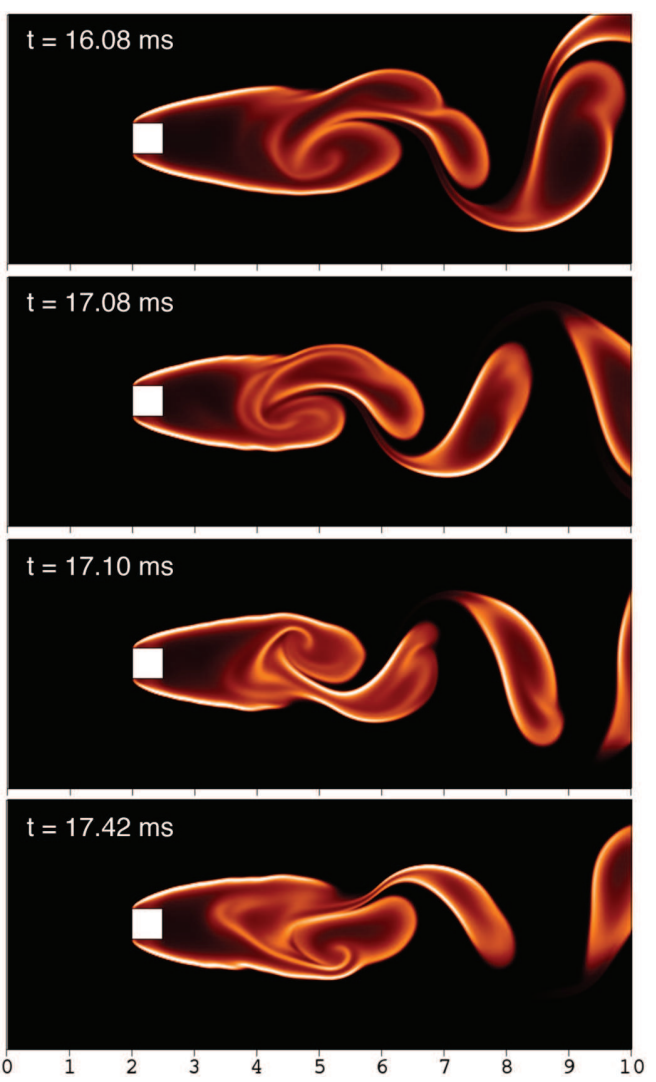

FigURE 7. Isocontours of heat release rate for hydrogen-air flames at $U=85 \mathrm{~m} / \mathrm{s}$. 

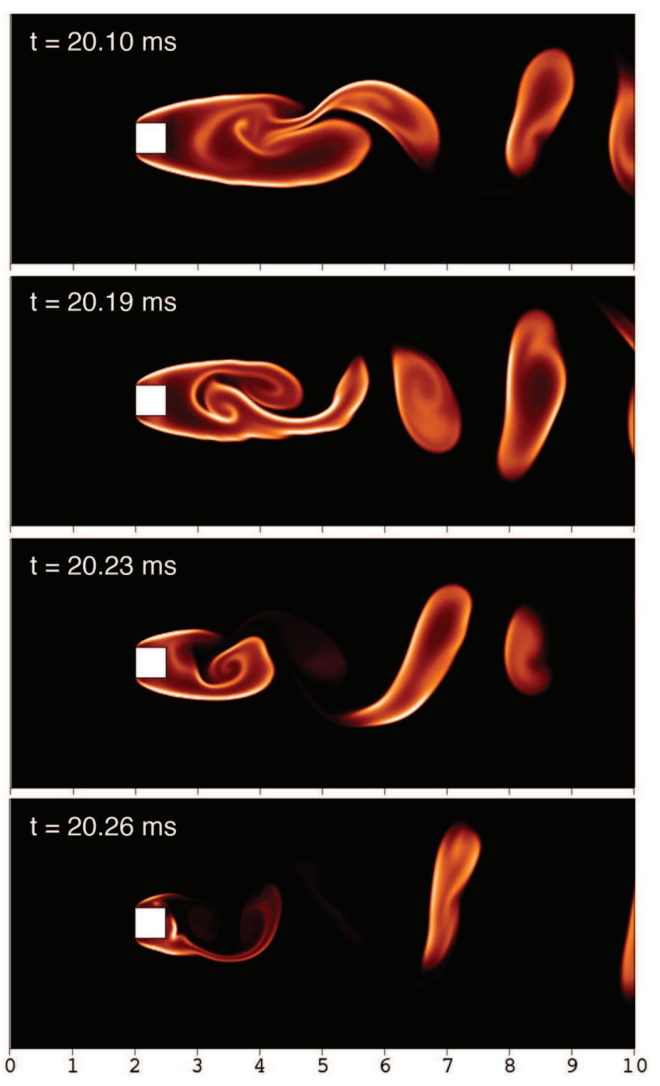

FiguRE 8. Isocontours of heat release rate for hydrogen-air flames at $U=85.2 \mathrm{~m} / \mathrm{s}$.

near leading edge of the bluff-body until $U=20 \mathrm{~m} / \mathrm{s}$. From $U=25$ to $40 \mathrm{~m} / \mathrm{s}$, the MF starts developing on the shear layer. From $U=48$ to $48.6 \mathrm{~m} / \mathrm{s}$, SVS appears. At $\mathrm{U}=48.6 \mathrm{~m} / \mathrm{s}$, the SVS mode is observed at the frequency of $6.8 \mathrm{kHz}$, as shown in Fig. 9(c, d). The recirculation zone behind a bluff-body periodically contracts and expands, consistent with the hydrogen-air flames. However, the transition to the AVS mode does not yet occur at this inflow velocity.

When the mean inflow velocity is slightly raised to $U=48.7 \mathrm{~m} / \mathrm{s}\left(R e_{D}=1410\right)$, the SVS mode continues in the beginning, and then the SVS-AVS-VST mode transition develops subsequently. The onset of the local extinction is also observed at the entrainment point in the AVS mode. After a few cycles of the transitions, the ultimate blow-off happens, while showing a gradual shrinkage of the attached flame behind a bluff-body. Figure 10 shows a progress of the blow-off event at $U=48.7 \mathrm{~m} / \mathrm{s}$. The VST mode starts showing up from $t=6.0 \mathrm{~ms}$, the attached flames on a bluff-body are gradually reduced in length, and then completely disappear around $t=6.5 \mathrm{~ms}$.

One of the key differences between hydrogen-air and syngas-air flames is noted just prior to the blow-off event. The attached flames in the syngas-air mixture are more robust than that in the hydrogen-air mixture. The first snapshots in Figures 8 and 10 are chosen as the flames under the similar topological hydrodynamic instability behavior. While the syngas-air flame still sustains itself after $0.16 \mathrm{~ms}$ (see the last snapshot in Fig. 10), the hydrogen-air flames are already hardly noticeable, as shown in the last image in Figure 8. This is consistent with the finding in our previous narrow channel study [12], where the attached flame kernel of syngas-air flame was more robust than hydrogen-air flame in the near blow-off condition. The robustness of the syngas-air flame was attributed to its lower sensitivity of heat release rate to the temperature fluctuation compare to the hydrogen-air flame [12]. 


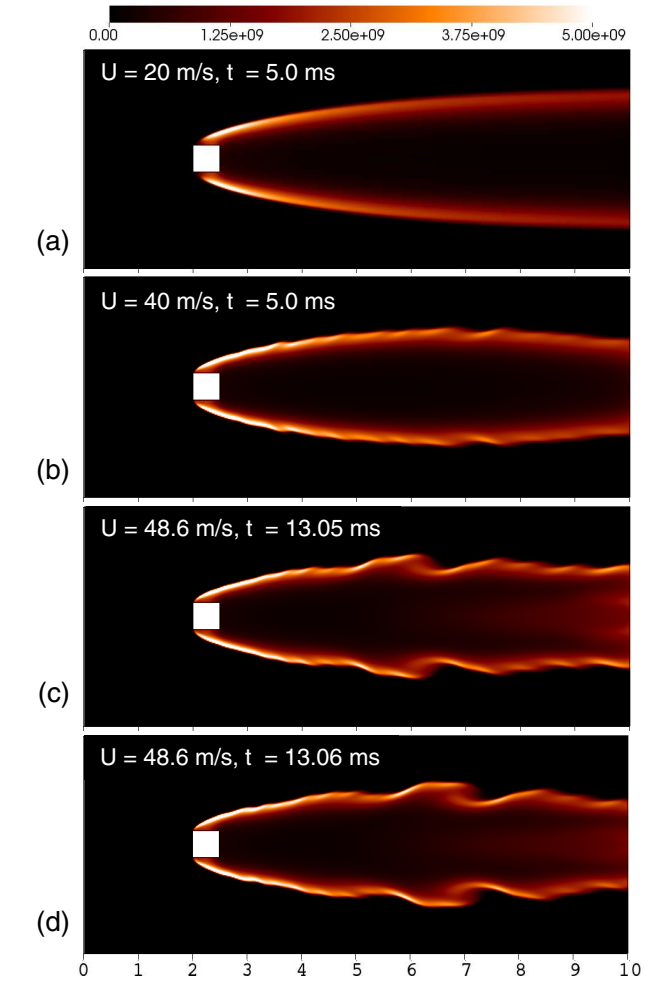

Figure 9. Isocontours of heat release rate for syngas-air flames: (a) $U=20 \mathrm{~m} / \mathrm{s}$, (b) $U=40 \mathrm{~m} / \mathrm{s}$, and $(\mathrm{c}, \mathrm{d}) U=48.6 \mathrm{~m} / \mathrm{s}$.

\subsubsection{Comparison of hydrogen-air vs. syngas-air flames}

To sum up the findings between hydrogen-air and syngas-air mixtures, the pathways of the flame instability from the steady to blow-off are presented schematically in Figure 11 as a function of the mean inflow velocity. The previous results $[12,13]$ are presented to compare with the present results. For the present results (Fig. 11b) in both hydrogen-air and syngas-air mixtures, flame instability is monotonically developed as the inflow velocity approaching blow-off limit. Stable flames are observed at the low velocity, and the flame undergoes symmetrical and asymmetrical instabilities as ramping up the mean inflow velocity. Once the asymmetrical instability is triggered, periodic transitions take place in which the three SVS-AVS-VST modes repeat. When the inflow velocity reaches the blow-off limit, after a few cycles of the transitions, the attached flame fails to sustain itself through the VST mode and results in the total blow-off. In both mixtures, the VST mode acts as a precursor of the ultimate blow-off, serving as a common behavior prior to complete extinction.

There are two major differences between two mixtures. First, the inflow velocities for the onset of the local extinction differ significantly, at $U=75 \mathrm{~m} / \mathrm{s}$ and $U=48.7 \mathrm{~m} / \mathrm{s}$ for hydrogen-air and syngas-air mixtures, respectively. Secondly, the flame dynamics from the onset of local extinction to blow-off are observed in a wider range of velocity (from $U=75$ to $85.2 \mathrm{~m} / \mathrm{s} ; \Delta U=10.2 \mathrm{~m} / \mathrm{s}$ ) for hydrogen-air mixture, whereas the range is much narrower (at $U=48.7 \mathrm{~m} / \mathrm{s} ; \Delta U=0.1 \mathrm{~m} / \mathrm{s}$ ) for the syngas-air mixture. These differences will be discussed in the subsequent section with the analysis of characteristic time scales based on the relevant quantities and the topological comparison.

Contrary to previous narrow channel studies $[12,13]$, in the absence of the confinement effect the flames were allowed to stabilize on the leading edge of the bluff-body at lower velocities, and near the blow-off limit exhibit a large level of lateral fluctuations to yield a more complicated pathway to blow-off with the transitions between SVS-AVS-VST modes. For the hydrogen-air flames, blow-off did not immediately occur after the onset of local 

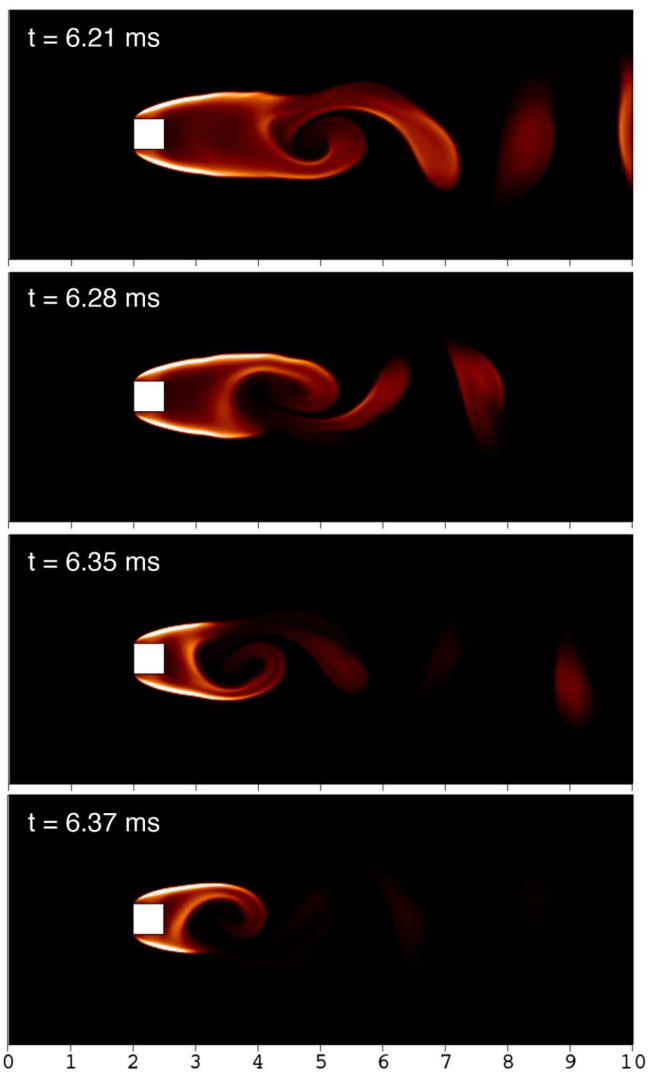

FiguRE 10 . Isocontours of heat release rate for syngas-air flames at $U=48.7 \mathrm{~m} / \mathrm{s}$.

(a)

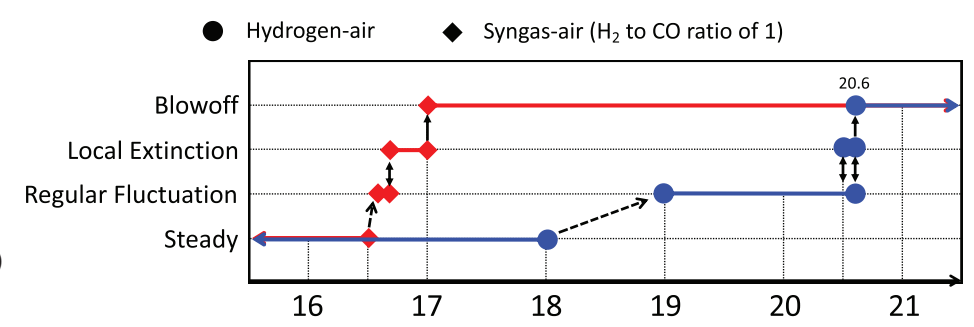

(b)

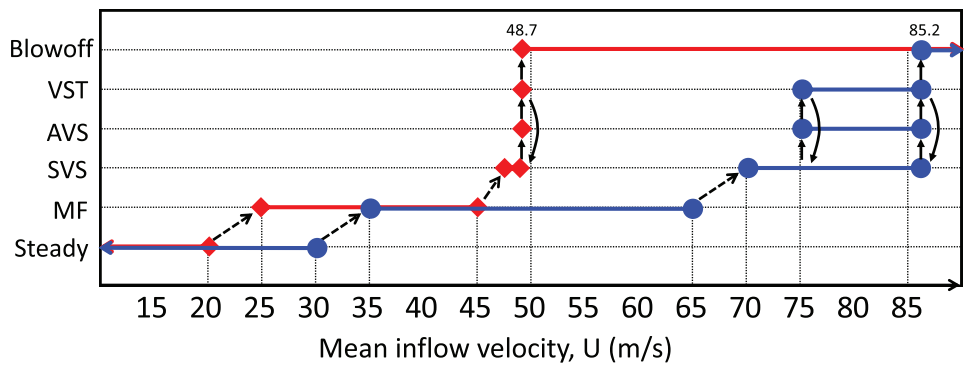

FiguRE 11. Diagrams of flame modes as increasing the mean inflow velocity until blow-off occurs in both hydrogen-air and syngas-air mixtures: (a) previous results [12, 13] and (b) present results. 
extinction, unlike our previous studies $[12,13]$. Although the syngas-air flames in this study was observed the blow-off event followed by the onset of local extinction as the previous studies [12, 13], the attached flame kernel of syngas-air mixture just prior to the blow-off event was still robust compared to that of hydrogen-air mixture, consistent with the findings in our previous study [12].

\subsection{Time scale analysis}

The previous narrow channel studies $[12,13]$ reported the onset of local extinction was determined by flame time scales, and this is the dominant factor to determine the ultimate extinction. Their finding was explained by the Damköhler number $(D a)$ correlations, which were successful in the scaling law for the blow-off limits. In the present study without the channel confinement, however, near-blow-off dynamics become more complicated, as the lateral motion becomes pronounced due to the absence of the flow confinement between the narrow channel. Therefore, characteristic time scales of both longitudinal and lateral motions are considered as the flow time scale.

Characteristic time scales and corresponding quantities for both hydrogen-air and syngas-air mixtures are investigated and summarized in Table 1 . The first columns for each mixture indicate the characteristic quantities and the time scales for the flow velocities at the onset of local extinction, and the additional columns for hydrogen-air mixture are for higher velocities associated with local extinction during the SVS-AVS-VST mode transitions. The local extinction was observed at the entrainment point of the fresh mixture in the AVS mode, which is near the end of the recirculation zone. The location of the local extinction was chosen according to the progress variable analysis over entire duration of the AVS mode. To develop a proper Damköhler number scaling, two flow time scales and two chemical time scales are considered. For the flow time scale, a longitudinal flow residence time, $\tau_{\text {res }}$, was defined as the ratio of the length from the leading edge of a bluff-body to a local extinction point $\left(x_{\text {ext }}\right)$ and the mean inflow velocity. For the lateral flow time scale, $\tau_{\text {lateral }}$, the observed oscillation time in lateral fluctuation was used. For the chemical time scales, the ignition delay time at the local extinction point, $\tau_{\mathrm{ig}}$, was calculated using composition of inflow mixtures and local temperature at the local extinction point probed from the solutions of numerical simulations. In addition, the nominal flame time scale, $\tau_{\text {flame }}$, was defined as the nominal flame thickness $\left(\alpha / S_{\mathrm{L}}\right)$ over the corresponding laminar flame speed $\left(S_{\mathrm{L}}\right)$. In the previous study [12], an alternative definition of the flame thickness $\left(\delta=\left(T_{\mathrm{b}}-T_{\mathrm{u}}\right) /(\mathrm{d} T / \mathrm{d} x)_{\max }\right)$ was also used, which resulted in $D a$ smaller by an order of magnitude. Therefore, only the nominal flame thickness was employed as the flame length scale as a generous measure. Combining the above flow and chemical time scales, a total of four $D a$ were defined as the ratio of flow and chemical time scale: two $D a_{\text {ig }}$ and two $D a_{\text {flame }}$.

Although both $D a_{\mathrm{ig}}$ and $D a_{\text {flame }}$ are in the same order of magnitude and show similar qualitative trends, $D a_{\mathrm{ig}}$ is considered the better choice for the present analysis in that it is consistently closer to unity than $D a_{\text {flame }}$. This is also in agreement wth the classic blow-off theory by Zukoski and Marble [23], in which the blow-off criterion was defined as the unity of Damköhler number based on flow residence time (the ratio of recirculation zone length and the physical velocity at the blow-off) and ignition delay time at the end of recirculation zone representing the local extinction point. This implies that the local extinction is associated with the failure of ignition of the reactant mixture. Using the ignition time scale, both $D a_{\text {ig }}$ numbers based on longitudinal residence time and lateral residence time are similar to each other, and account for the approach to blow-off showing departure from unity as the hydrogen-air flame approaches to the blow-off limit. The lateral fluctuation is due to the AVS, the frequency of which is characterized using the Strouhal number, $S t=f D / U$. Considering that $S t$ is found nearly constant within the range of inflow velocities of the present study $\left(R e_{D}\right.$ from 1990 to $2260), U$ scales linearly with $f$. This implies that the increase in the inflow velocity causes not only a decrease in the longitudinal residence time, but also a decrease in the lateral residence time. Therefore, it is difficult to identify which choice of $D a_{\text {ig }}$ is more appropriate as the flame approaches blow-off limit.

In the previous narrow channel studies [12, 13], the onset of local extinction led to the ultimate blow-off upon a slight increase in the inflow velocity. In the present study, however, the local extinction does not immediately lead to the total extinction, at least for the hydrogen-air mixture. There is more complex dynamics in the hydrogen-air mixture from the onset of local extinction to the blow-off limit which corresponds to a range of the 
TABlE 1. Characteristic time scales and quantities for both hydrogen-air and syngas-air mixtures at pressure of 1 atm.

\begin{tabular}{|c|c|c|c|c|}
\hline Mixture & \multicolumn{3}{|c|}{ Hydrogen-air } & Syngas-air \\
\hline $\begin{array}{l}\Phi \\
T_{\text {in }}(\mathrm{K}) \\
\rho_{\mathrm{u}} / \rho_{\mathrm{b}} \\
S_{\mathrm{L}}(\mathrm{m} / \mathrm{s})\end{array}$ & & $\begin{array}{l}4.91 \\
0.49\end{array}$ & & $\begin{array}{l}0.5 \\
300 \\
5.15 \\
0.30\end{array}$ \\
\hline $\begin{array}{l}U(\mathrm{~m} / \mathrm{s}) \\
R e_{D} \\
x_{\text {ext }}(\mathrm{mm})\end{array}$ & $\begin{array}{l}75 \\
1990 \\
3.02\end{array}$ & $\begin{array}{l}80 \\
2120 \\
2.84\end{array}$ & $\begin{array}{l}85.2 \\
2260 \\
1.90\end{array}$ & $\begin{array}{l}48.7 \\
1410 \\
3.04\end{array}$ \\
\hline $\begin{array}{l}\tau_{\text {res }}\left(x_{\text {ext }} / U\right)(\mathrm{ms}) \\
\tau_{\text {lateral }}(\text { observed })(\mathrm{ms})\end{array}$ & $\begin{array}{l}0.040 \\
0.040\end{array}$ & $\begin{array}{l}0.036 \\
0.035\end{array}$ & $\begin{array}{l}0.022 \\
0.030\end{array}$ & $\begin{array}{l}0.062 \\
0.061\end{array}$ \\
\hline $\begin{array}{l}\tau_{\text {ig }}\left(T \text { at } x_{\text {ext }}\right)(\mathrm{ms}) \\
D a_{\text {ig }}\left(\tau_{\text {res }} / \tau_{\text {ig }}\right) \\
D a_{\text {ig }}\left(\tau_{\text {lateral }} / \tau_{\text {ig }}\right)\end{array}$ & $\begin{array}{l}0.064 \\
0.63 \\
0.63\end{array}$ & $\begin{array}{l}0.070 \\
0.51 \\
0.50\end{array}$ & $\begin{array}{l}0.067 \\
0.33 \\
0.45\end{array}$ & $\begin{array}{l}0.103 \\
0.60 \\
0.59\end{array}$ \\
\hline $\begin{array}{l}\tau_{\text {flame }}\left(\alpha / S_{\mathrm{L}}^{2}\right)(\mathrm{ms}) \\
D a_{\text {flame }}\left(\tau_{\text {res }} / \tau_{\text {flame }}\right) \\
D a_{\text {flame }}\left(\tau_{\text {lateral }} / \tau_{\text {flame }}\right)\end{array}$ & $\begin{array}{l}0.151 \\
0.26 \\
0.26\end{array}$ & $\begin{array}{l}0.151 \\
0.24 \\
0.23\end{array}$ & $\begin{array}{l}0.151 \\
0.15 \\
0.20\end{array}$ & $\begin{array}{l}0.332 \\
0.19 \\
0.18\end{array}$ \\
\hline
\end{tabular}
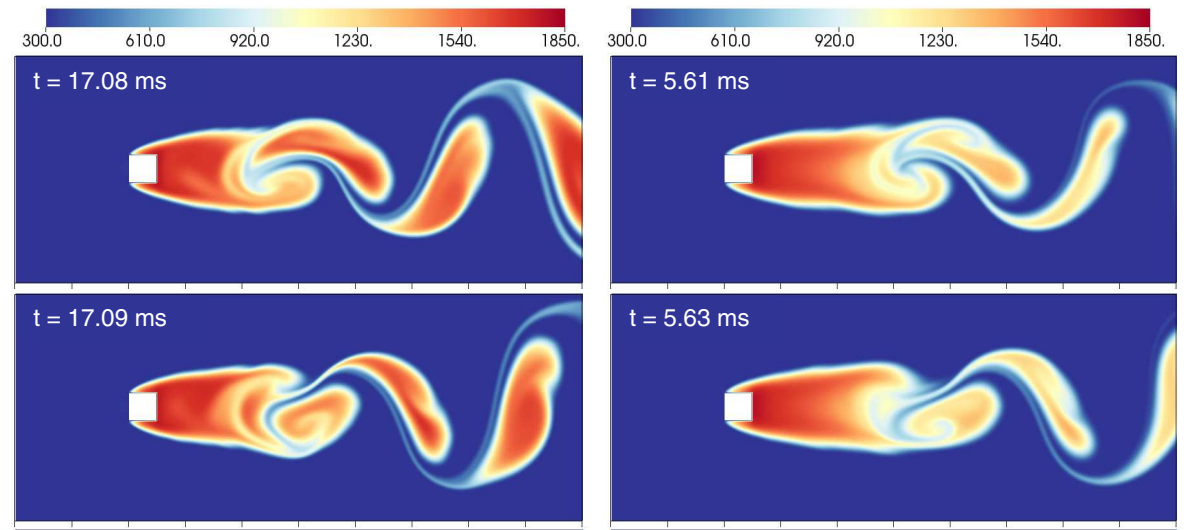

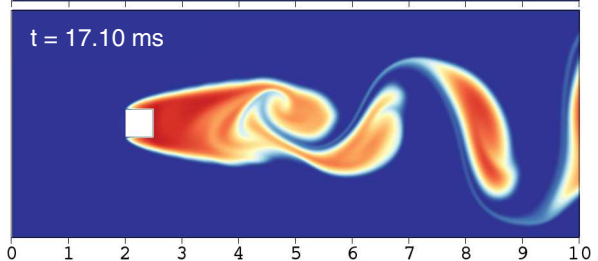

(a)

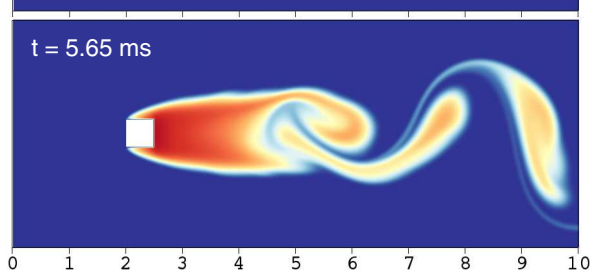

(b)

FiguRE 12. Isocontours of temperature distributions: (a) hydrogen-air at $U=85 \mathrm{~m} / \mathrm{s}$ and (b) syngas-air at $U=48.7 \mathrm{~m} / \mathrm{s}$.

mean inflow velocity $(U)$ from 75 to $85.2 \mathrm{~m} / \mathrm{s}$. To further investigate the hydrogen flame dynamics, differences in the local flame-flow interaction dynamics between hydrogen-air and syngas-air flames are examined.

Figure 12 shows the temperature distributions in a series of instantaneous snapshots to visualize the sequence of lateral fluctuation in (a) hydrogen-air and (b) syngas-air mixtures. The comparison starts from the moment 


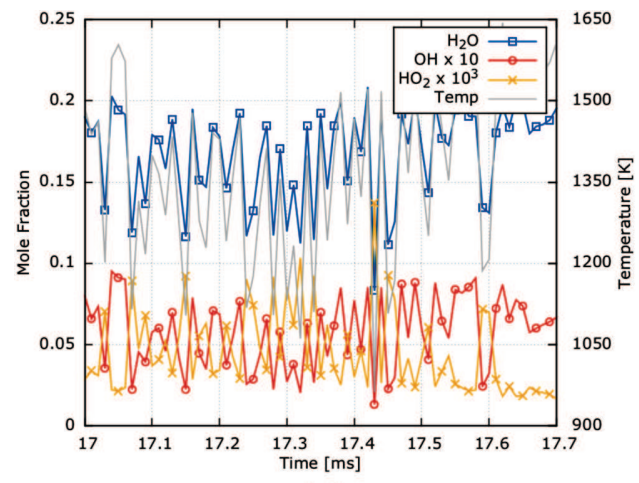

(a)

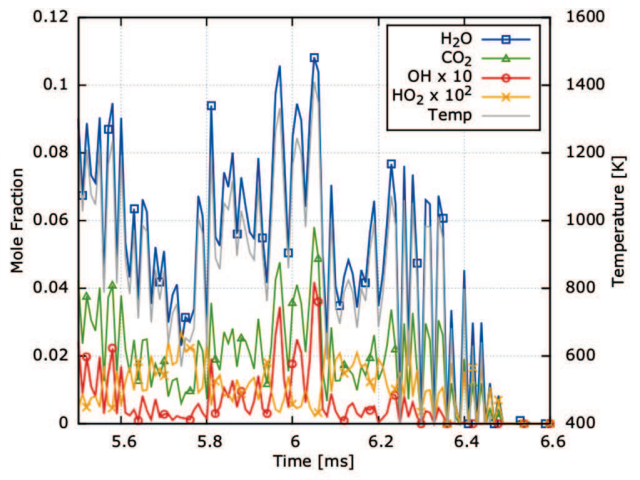

(b)

FiguRE 13. Temporal evolutions of principal species mole fractions for several periods of fluctuations, probed at the local extinction points: (a) hydrogen-air at $U=85 \mathrm{~m} / \mathrm{s}$ and (b) syngas-air at $U=48.7 \mathrm{~m} / \mathrm{s}$.

of flames under similar hydrodynamic instability (see in the first snapshots of Fig. 12), followed by subsequent temporal events. Both flames have a hot recirculation zone behind the bluff-body, and it is confined by the rolled-up AVS. Although both flames are showing the VST mode, the hydrogen-air flame continues to be in the VST mode for a few more cycles. However, the syngas-air flame immediately reached the total blow-off after a few milliseconds. The observed time scales of lateral fluctuation, $\tau_{\text {lateral }}$, are 0.03 and $0.06 \mathrm{~ms}$ in hydrogen-air and syngas-air flames, respectively. Therefore, the hydrodynamic parameters (the mean inflow velocity and the lateral time scale) appear to be more unfavorable (faster velocity and shorter time scale) for the hydrogen-air than those for the syngas-air flames, yet the hydrogen-air flames are found to be more resilient to extinction during the AVS mode. A closer examination of the hydrogen-air flames reveals that an isolated hot pocket is immediately entrained into the local extinction area, as seen in the second snapshots in Figure 12a. On the other hand, the isolated gas pocket is lower in temperature and relatively further detached from the extinction zone, thereby failing to re-ignite the reactants. Therefore, it appears that the fast lateral fluctuations in the hydrogenair flames help sustain the combustion due to the re-ignition mechanism by the product pocket entrainment. This observation is further supported by the fact that the ignition delay time at the edge of the pocket entrainment is much shorter for the hydrogen-air mixtures compared to the syngas-air counterpart. The re-ignition around the local extinction area is also supported by the observation of the phase shift of $\mathrm{HO}_{2}$, which is an important marker species for ignition, along the extinction phases based on the temperature fluctuation, as shown in Figure 13, which was also reported in our previous study [12].

The overarching summary of the present study of unconfined bluff-body flame stabilization is that the larger extent of the lateral movements resulting from the vortex shedding patterns shifts the fundamental flame stabilization mechanism from local extinction problem in the previous studies $[12,13]$ to the re-ignition problem around the local extinction area, making the blow-off criterion re-aligned with the Zukoski-Marble theory [23]. That is, the blow-off is determined by how quickly the product gas pocket becomes entrained into the extinguished mixture zone, combined with how quickly the local reactant mixture can ignite at the corresponding thermo-chemical conditions. As such the Damköhler number based on the ignition delay time is found to be a better representation of the reactive time scales to characterize the blow-off behavior of unconfined flames behind a bluff-body.

\section{Conclusion}

Two-dimensional direct numerical simulations were conducted to investigate blow-off dynamics in lean premixed hydrogen-air and syngas $\left(\mathrm{H}_{2}\right.$ to $\mathrm{CO}$ ratio of 1 )-air mixtures under a reduced confinement channel with 
a meso-scale bluff-body. Flame/flow dynamics were investigated as increasing the mean inflow velocity until blow-off limit.

Contrary to the previous studies $[12,13]$, the absence of the flow confinement resulted in a significantly larger level of fluctuations both longitudinally and laterally, thus leading to a more complicated pathways to blow-off while undergoing transitions between several distinct modes of flame instabilities towards the blow-off limit. For both mixtures, the observed five distinct flame modes were categorized, such as steadily stable (steady), MF SVS, AVS, and VST modes. The flame modes were monotonically enhanced from symmetric (SVS) to asymmetric structures (AVS-VST). At the near-blow-off condition, flames underwent the periodic transition between SVS mode and AVS-VST mode and then proceeded to the ultimate blow-off. To our knowledge, this is the first DNS result reporting such behavior.

In time scale analysis, both the onset of local extinction and blow-off limit were found to be better correlated with the Damköhler number based on ignition delay time, consistent with the earlier theory [23]. The observed shift in the fundamental mechanism for flame stabilization results from the increased lateral fluctuations due to the AVS, leading to a combined effects of hot gas entrainment and auto-ignition of the extinction zone. Due to the complex sequence of symmetric and AVS modes, however, further investigation is needed in order to systematically isolate different fluid dynamic sub-processes in affecting larger scale flame stabilization.

The size of the bluff body was at millimeter scale for the present study. As long as the ignition time scale governs the flame dynamics at near-blowoff regime, the observation at this scale would still be valid for larger scale bluff bodies, because the competition between the flow time scale for the supply of reactant at the end of the heat pocket (i.e. the recirculation area) and the chemical time scale for the ignition delay does not depend on the absolute scales. However, this hypothesis needs to be verified in the future study for laboratory-scale bluff-bodies.

Acknowledgements. The research reported in this publication was supported by funding from King Abdullah University of Science and Technology (KAUST) and computing cluster provided by KAUST Supercomputing Laboratory (KSL). The second author was partly supported by Advanced Research Center Program (2013R1A5A1073861) and Basic Science Research Program (2017R1A2B4003327) through the National Research Foundation of Korea.

\section{REFERENCES}

[1] A.A. Chaparro and B.M. Cetegen, Blowoff characteristics of bluff-body stabilized conical premixed flames under upstream velocity modulation. Combust. Flame 144 (2006) 318-335.

[2] J.H. Chen, A. Choudhary, B. de Supinski, M. DeVries, E.R. Hawkes, S. Klasky, W.K. Liao, K.L. Ma, J. Mellor-Crummey, N. Podhorszki, R. Sankaran, S. Shende and C.S. Yoo, Terascale direct numerical simulations of turbulent combustion using S3D. Comput. Sci. Discov. 2 (2009) 015001.

[3] I. Glassman, R.A. Yetter and N.G. Glumac, Combustion. Academic Press (2014).

[4] J. Hertzberg, I. Shepherd and L. Talbot, Vortex shedding behind rod stabilized flames. Combust. Flame 86 (1991) 1-11.

[5] E. Hodzic, E. Alenius, C. Duwig, R. Szasz, L. Fuchs, A large eddy simulation study of bluff body flame dynamics approaching blow-off. Combust. Sci. Technol. 189 (2017) 1107-1137.

[6] K.S. Kedia and A.F. Ghoniem, The anchoring mechanism of a bluff-body stabilized laminar premixed flame. Combust. Flame 161 (2014) 2327-2339.

[7] K.S. Kedia, A. F. Ghoniem, The blow-off mechanism of a bluff-body stabilized laminar premixed flame. Combust. Flame $\mathbf{1 6 2}$ (2015) 1304-1315.

[8] R.J. Kee, G. Dixon-Lewis, J. Warnatz, M.E. Coltrin and J.A. Miller, A FORTRAN computer code package for the evaluation of gas-phase, multicomponent transport properties. Technical Report SAND86-8246, Sandia National Laboratories (1986).

[9] R.J. Kee, F.M. Rupley and J.A. Miller, CHEMKIN-II: A FORTRAN chemical kinetics package for the analysis of gas-phase chemical kinetics. Technical Report SAND89-8009, Sandia National Laboratories (1989).

[10] Y.J. Kim, B.J. Lee and H.G. Im, Near-blowoff dynamics of lean premixed flames stabilized on a meso-scale bluff body, 54th AIAA Aerospace Sciences Meeting (2016) 0956.

[11] K. Kundu, D. Banerjee and D. Bhaduri, Theoretical analysis on flame stabilization by a bluff-body. Combust. Sci. Technol. 17 (1977) 153-162.

[12] B.J. Lee and H.G. Im, Dynamics of bluff-body-stabilized lean premixed syngas flames in a meso-scale channel. Proc. Combust. Inst. 36 (2017) 1569-1576.

[13] B.J. Lee, C.S. Yoo and H.G. Im, Dynamics of bluff-body-stabilized premixed hydrogen/air flames in a narrow channel. Combust. Flame 162 (2015) 2602-2609. 
[14] J. Li, Z. Zhao, A. Kazakov and F.L. Dryer, An updated comprehensive kinetic model of hydrogen combustion. Int. J. Chem. Kinet. 36 (2004) 566-575.

[15] J. Li, Z. Zhao, A. Kazakov, M. Chaos, F.L. Dryer and J.J. Scire, A comprehensive kinetic mechanism for $\mathrm{CO} \mathrm{CH}_{2} \mathrm{O}$, and $\mathrm{CH}_{3} \mathrm{OH}$ combustion. Int. J. Chem. Kinet. 39 (2007) 109-136.

[16] S.J. Shanbhogue, S. Husain, T. Lieuwen, Lean blowoff of bluff body stabilized flames: Scaling and dynamics. Proc. Combust. Inst. 35 (2009) 98-120.

[17] K. Suzuki and H. Suzuki, Instantaneous structure and statistical feature of unsteady flow in a channel obstructed by a square rod. Int. J. Heat Fluid Flow 15 (1994) 426-437.

[18] H. Suzuki, Y. Inoue, T. Nishimura, K. Fukutani and K. Suzuki, Unsteady flow in a channel obstructed by a square rod (crisscross motion of vortex). Int. J. Heat Fluid Flow 14 (1993) 2-9.

[19] S.G. Tuttle, S. Chaudhuri, K.M. Kopp-Vaughan, T.R. Jensen, B.M. Cetegen, M.W. Renfro and J.M. Cohen, Lean blowoff behavior of asymmetrically-fueled bluff body-stabilized flames. Combust. Flame 160 (2013) 1677-1692.

[20] G.C. Williams, H. Hottel and A.C. Scurlock, Flame stabilization and propagation in high velocity gas streams. Symp. Combust. Flame Explos. Phenom. 3 (1948) 21-40.

[21] C.S. Yoo and H.G. Im, Characteristic boundary conditions for simulations of compressible reacting flows with multidimensional, viscous and reaction effects. Combust. Theory Model. 11 (2007) 259-286.

[22] C.S. Yoo, Y. Wang, A. Trouvé and H.G. Im, Characteristic boundary conditions for direct simulations of turbulent counterflow flames. Combust. Theory Model. 9 (2005) 617-646.

[23] E.E. Zukoski and F.E. Marble, Experiments concerning the mechanism of flame blowoff from bluff bodies. Proc. Gas Dyn. Symp. Aerothermochem. (1956) 205-210. 